\title{
A Prospective Assessment of Psychosocial Factors Among Bariatric Versus Non-bariatric Surgery Candidates
}

\author{
Thomas Rutledge $\cdot$ Sarah Adler $\cdot$ Raquel Friedman
}

Published online: 25 September 2010

(C) The Author(s) 2010. This article is published with open access at Springerlink.com

\begin{abstract}
Background Psychological factors are considered potential contraindicators to bariatric surgery, but inconsistently predict surgical outcomes. We examined biomedical and psychosocial predictors of future bariatric candidacy in a population of veterans enrolling in a multidisciplinary weight management program.

Methods Ninety-five obese veterans meeting bariatric surgery eligibility criteria participating in a weight control intake class from 2007 to 2008 completed the MOVE!23 questionnaire to assess biomedical, psychiatric, social, and eating behavior factors. Twenty-five patients from this cohort completed or obtained approval for bariatric surgery during the next 2 years of follow-up.

Results Patients progressing to bariatric candidacy over follow-up differed from non-bariatric patients in multiple areas, including reporting significantly lower rates of depression (28\% versus $48.7 \%$, respectively; $p=0.04)$ and
\end{abstract}

\author{
T. Rutledge $(\bowtie)$ \\ Medical Center, \\ 3350 La Jolla Village Drive, \\ San Diego, CA 92161, USA \\ e-mail: Thomas.Rutledge@va.gov \\ T. Rutledge \\ Department of Psychiatry, UC San Diego, \\ San Diego, CA, USA \\ S. Adler \\ Department of Psychiatry, Stanford University, \\ Stanford, CA, USA \\ R. Friedman \\ UC San Diego, \\ San Diego, CA, USA
}

Psychology Service 116B, VA San Diego Healthcare System, smoking ( $4 \%$ versus $16 \%$; $p=0.05$ ), better self-rated health (e.g., $28 \%$ versus $10.7 \%$ rating themselves as in excellent or very good health), and averaged $50 \%$ fewer cardiovascular risk factors $(p=0.01)$. Bariatric patients also rated themselves as significantly faster eaters $(p=.03)$ and as having higher rates of obsessive compulsive disorder (OCD; 28\% versus 7\%; $p=0.04$ ). Depression and OCD status predicted patients going on to bariatric candidacy independent of body mass index (BMI), biomedical status, and demographic factors.

Conclusions Our results suggest that many of the commonly cited psychosocial contraindicators to bariatric surgery are already lower in patients considered for surgery relative to BMI equivalent treatment-seeking peers not approved for surgery. These differences may help explain inconsistent relationships between psychosocial factors and bariatric surgery outcomes.

Keywords Bariatric surgery · Psychological factors . Veteran

The use of psychological assessments among pre-bariatric patients presents a divide between research and practice. Surveys of bariatric surgery clinics indicate that the majority include a psychological assessment as part of the pre-bariatric evaluation $[1,2]$. Guidelines from major bariatric surgery societies, such as the American Society of Metabolic and Bariatric Surgery, also recommend psychological evaluations for pre-bariatric patients as standard of care [3]. The rationale presented within these recommendations is that psychiatric concerns in bariatric candidates are common [4] and that the identification of these factors can improve the selection process for bariatric 
surgery patients, enhance weight-related surgical outcomes, and reduce the incidence of complications such as weight regain and adherence difficulties [5, 6]. Given the rapid growth in the number of bariatric procedures performed annually in the USA [7], an $80 \%$ compliance rate with psychological assessment recommendations suggests an enormous annual investment for mental health providers performing these evaluations.

However, there is a lack of compelling statistical evidence to support that these assessments are accomplishing their intended goals. Across at least five [8-12] reviews of the pre-bariatric psychological assessment literature published in the past decade, none suggest that psychosocial factors consistently predict weight-related surgery outcomes.

This disparity between clinical guidelines and empirical evidence is troubling. On one hand, if the lack of empirical support is indicative of a lower than perceived clinical value for pre-bariatric psychological assessments, then the assessment process requires critical evaluation [13]. Alternatively, it is also possible that existing research on the subject suffers from shortcomings that underestimate the impact of psychological factors on post-surgical outcomes.

One such shortcoming is that prior research on this topic focuses almost exclusively on patients actually undergoing bariatric surgery. Patients receiving bariatric surgery are not a random group. Even among body mass index (BMI)eligible obese patients seeking surgery, they must meet cardiovascular safety criteria; obtain approval from the surgeon, mental health provider, and possibly other healthcare team members; and sometimes show evidence of behavioral change through such standards as a pre-surgery $5 \%$ weight loss [14]. The result, in theory, is a surgery sample comprised of healthier patients with evidence of stable mental health and behavioral compliance.

There are unintended effects, however, that make the goals of the selection process difficult to examine. For example, patients not approved for bariatric surgery are not available for comparison, making it impossible to verify whether any identified concerns would have affected surgery outcomes. Similarly, the process of pursuing and obtaining approval for bariatric surgery acts to homogenize the sample on both physical and psychiatric dimensions. Those with poorer medical and mental health are eliminated from consideration, with the statistical ramification being reduced variability for the remaining sample. Because constraints in variability act to reduce the magnitude of relationships between variables, understanding the extent to which bariatric candidates may differ from non-candidates is important to interpreting previous research on psychosocial predictors of bariatric surgery outcomes [15].

The presence of selection factors among bariatric surgery patients cannot be effectively examined at the level of patients considered for surgery, by which point many patients have been eliminated from the process. Instead, a prospective sample is necessary, wherein a cohort of surgery-eligible patients is followed over time to assess how those pursuing bariatric surgery may differ from those not pursuing or not approved for surgery. We assessed this topic in a Veteran population completing a standardized education class series, a subset of whom went on to obtain or be approved for bariatric surgery over a 2-year follow-up.

\section{Method}

\section{Participants}

The study sample comprised 95 consecutive adult veterans completing an intake class required for entry into the Managing Overweight/Obesity in Veterans Everywhere (MOVE) program and weight control clinic services of the VA San Diego Healthcare System (VASDHS) between September of 2007 and August of 2008. Participants in the current study had either a $\mathrm{BMI} \geq 40$ or $\mathrm{BMI} \geq 35$ along with a major weight-related comorbidity (e.g., diabetes, cardiovascular disease, sleep apnea, hypertension, dyslipidemia), age $\leq 70$, spoke English as their primary language, and were determined by their primary care provider to be safe for participating in a program involving diet and exercise lifestyle changes.

As part of the intake class, participants completed physical height and weight measurements, a detailed questionnaire concerning their physical and mental health, eating and exercise patterns, diet history, and psychosocial circumstances impacting weight loss. Completion of the intake and MOVE classes is required in the VASDHS for patients to be considered for bariatric surgery, ensuring that patients complete a standardized process through weight control clinic services. Patients are queried regarding their interest in bariatric surgery at the conclusion of the MOVE classes. The intake class and subsequent weight control clinic services (including bariatric surgery) are also free of charge to veterans completing the initial paperwork, facilitating equal access to services for all program participants. Following the intake class, we subsequently followed participants through the VASDHS Computerized Patient Record System regarding surgical, medication, and behavioral weight loss services through the weight control clinic. For the current paper, we tracked participants regarding consideration for bariatric surgery through June of 2010. Candidates were identified as either those completing bariatric surgery through the VASDHS facility or approved for and awaiting surgery through the weight control clinic as part of surgery consideration. University of California, San Diego and VASDHS IRB approval was obtained as part of the study completion. 


\section{MOVE!23 Questionnaire}

As part of the intake class, participants completed the MOVE! 23 questionnaire, a 23-item instrument developed by the National Center for Health Promotion and Disease Prevention (NCP) that assesses a broad range of information including demographics, medical and psychiatric diagnoses, eating patterns, physical activity, and psychosocial factors that may impact weight management [16]. Through use of the national MOVE! Website, data from the MOVE!23 questionnaire result in a detailed four- to six-page summary describing individualized factors potentially affecting weight management for the participant. The MOVE!23 questionnaire requires an estimated 11 min to complete (see Appendix for the complete measure). Using the patient's MOVE!23 unique extraction code, we retrieved each complete questionnaire result for data analyses.

\section{Statistical Analyses}

The 95 study participants resulted from a total of 127 intake class enrollees. This total represented $74.8 \%$ of the intake class patients. Study analyses comprised descriptive statistics, independent group $t$ tests, and chi-square analyses comparing bariatric surgery versus non-surgery participants. Factors on which we completed group comparisons included demographic (age, ethnicity, gender), biomedical (weight and BMI, history of heart disease, stroke, and major cardiovascular risk factors), and psychosocial variables cited in previous literature as common contraindicators to bariatric surgery (psychiatric diagnoses, substance use, binge eating, weight loss expectations, eating patterns, etc.). After adjusting for age and BMI, we entered factors identified as significantly different between bariatric surgery versus nonsurgery patients at the bivariate level in a logistic regression to assess which of the latter factors most effectively predicted subsequent surgery candidacy. The criterion for statistical significance was set at $p<0.05$. All calculations were performed using SPSS 17.0 (www.SPSS.com).

\section{Results}

Among 95 eligible patients completing MOVE! intake classes during the observation period, 25 went on to bariatric surgery candidacy through the weight control clinic by the end of the study follow-up. Table 1 summarizes biomedical data comparing the bariatric versus nonbariatric patients. The latter values showed a general pattern for bariatric candidates carrying a lower chronic disease burden. Patients going on to bariatric candidacy had significantly lower self-reported rates of smoking (15.7\% versus $4.0 \%$ for non-bariatric versus bariatric patients, respectively, $p=0.03)$ and heart disease $(15.7 \%$ versus $4.0 \%$, respectively, $p=0.04$ ). Comparing the patient groups on a composite cardiovascular risk variable (comprised of stroke and heart disease history, diabetes, high cholesterol, smoking, hypertension, and family history of heart disease) showed that surgery patients averaged significantly fewer cardiovascular risk indicators $($ means $=2.1$ (1.4) versus 1.4 (1.2) for non-surgery versus surgery patients, respectively, $p=0.01$ ). Patient groups did not differ on demographic factors, body weight, or BMI indices.

Table 2 compares the patient groups on psychiatric and eating behavior pattern factors. Bariatric patients rated their health as significantly better (e.g., $28.0 \%$ versus $10.7 \%$ of the later bariatric surgery versus non-surgery candidates rated their health as either excellent or very good, $p=0.001$ ). Slightly more than $1 / 4$ of the bariatric candidate group endorsed depression (28\%) versus approximately half of the non-surgery candidate group $(48.7 \%, p=0.04)$. Bariatric candidates rated their eating speed as significantly faster (e.g., $64.0 \%$ versus $44.0 \%$ of the surgery versus nonsurgery group, respectively, rated themselves as a "fast"
Table 1 Demographic and biomedical factors among bariatric surgery and non-surgery candidates $(N=95)$
${ }^{*} p<0.05$

\begin{tabular}{lcc}
\hline Variable & Non-surgery patients $(n=70)$ & Surgery candidates $(n=25)$ \\
\hline Age (mean [standard deviation]) & $53.6(10.9)$ & $51.3(8.7)$ \\
BMI & $42.0(6.0)$ & $42.0(6.0)$ \\
BMI $\geq 40(\%)$ & 67.1 & 56.0 \\
Weight (lb) & $277.7(47.5)$ & $284.8(50.8)$ \\
Race (non-Caucasian) (\%) & 41.4 & 40.0 \\
Gender (female) (\%) & 25.7 & 16.0 \\
Diabetic (\%) & 42.9 & 24.0 \\
Hyperlipidemia (\%) & 54.3 & $32.0^{*}$ \\
Hypertension (\%) & 68.6 & 60.0 \\
Smoker (\%) & 15.7 & $4.0^{*}$ \\
Heart disease (\%) & 15.7 & $4.0^{*}$ \\
\hline
\end{tabular}


Table 2 Psychosocial factors and eating behaviors among bariatric surgery and non-surgery candidates $(N=95)$

PTSD post-traumatic stress disorder

${ }^{*} p<0.05$

\begin{tabular}{lll}
\hline Variable & $\begin{array}{l}\text { Non-surgery } \\
\text { patients }(n=70)\end{array}$ & $\begin{array}{l}\text { Surgery candidates } \\
(n=25)\end{array}$ \\
\hline $\begin{array}{l}\text { Self-rated health (excellent/very } \\
\text { good/good/fair/poor) (\%) }\end{array}$ & $2.7 / 8.0 / 36.0 / 38.7 / 14.7$ & $8.0 / 20.0 / 44.0 / 20.0 / 8.0^{*}$ \\
PTSD (\%) & 31.4 & 20.0 \\
Depression (\%) & 48.7 & $28.0 *$ \\
Anxiety (\%) & 31.4 & 32.0 \\
Binge eating at least 1× week (\%) & 82.3 & 88.0 \\
Substance abuse (\%) & 3.0 & 0.0 \\
Schizophrenia (\%) & 3.0 & 0.0 \\
Obsessive compulsive disorder (\%) & 7.0 & $28.0 *$ \\
Bipolar (\%) & 3.0 & 4.0 \\
Eating speed (slow/medium/fast) (\%) & $6.7 / 49.3 / 44.0$ & $0.0 / 36.0 / 64.0 *$ \\
12-month weight loss expectation & $4.0 / 16.0 / 53.3 / 22.7 / 4.0$ & $12.0 / 8.0 / 36.0 / 36.0 / 8.0$ \\
$\quad(0-10 / 10-25 / 25-50 / 50-100 / 100+)(\%)$ & $8.6(1.7)$ & $9.1(2.0)$ \\
Confidence in weight loss (0-10 scale) & $7.8(2.5)$ & $8.2(2.8)$ \\
Importance of weight loss (0-10 scale) & & \\
\hline
\end{tabular}

eater, $p=0.03$ ) and also reported higher rates of obsessive compulsive disorder (OCD; $p=0.04$ ). Rates of other common psychiatric contraindicators to bariatric surgery (e.g., substance abuse, schizophrenia, bipolar) affected less than $5 \%$ of the sample. Motivation and expectations for 12month weight loss were high among patients in both groups, with $80 \%$ of both groups reporting an expectation of $25+$ pound weight loss in the subsequent year. Binge eating rates exceeded $80 \%$ in both patient groups.

\section{Predicting Bariatric Candidacy from Intake Class Status}

In a logistic regression model predicting bariatric candidacy, we entered factors identified as significantly differing at the bivariate level between the patient groups (i.e., smoking status, heart disease, depression, OCD, self-rated health, and eating speed), including age and BMI as covariates. Only depression and OCD remained reliable predictors in this model. Bariatric candidates were less likely to endorse depression during the intake class $(\mathrm{OR}=0.21,95 \%$ $\mathrm{CI}=0.05-0.85)$ and more likely to endorse $\mathrm{OCD}(\mathrm{OR}=3.6$, $95 \% \mathrm{CI}=1.5-6.4)$ relative to non-surgery candidates.

\section{Discussion}

In this paper, we employed a prospective approach to examine factors associated with bariatric surgery candidacy. Tracking a group of 95 veterans meeting bariatric surgery eligibility criteria and enrolling in a multidisciplinary, lifestyle-based weight management program (MOVE) at a large VA medical center, we followed patients over an 18-24-month period to identify biomedical and psychosocial factors differentiating eventual bariatric surgery candidates versus non-surgery candidates during this period. Our results supported the hypothesis that differences exist between bariatric surgery and non-surgery patients and that these differences are present well before the stage of pre-surgery psychological evaluations. Although similar in demographic and weight-related variables, the subset of patients later completing bariatric surgery showed evidence of better cardiovascular health and higher functioning on several psychiatric dimensions commonly considered as potential contraindicators to surgery in pre-bariatric psychological evaluations (e.g., lower depression and smoking rates). Bariatric patients also endorsed higher rates of OCD and greater tendencies to engage in selfperceived fast eating behavior. In addition, psychological factors (depression and OCD) proved to be the best predictors of future bariatric candidacy, with those later pursuing surgery characterized as having lower self-reported rates of depression and higher rates of OCD.

To the extent that these results generalize to other bariatric surgery-eligible samples, an implication is that patients presenting for pre-bariatric psychological evaluations may differ in a variety of ways from patients otherwise matched on BMI. Although it is estimated that approximately $15 \%$ of bariatric candidates are declined for consideration due to psychosocial reasons [17], most of the most common psychosocial factors cited as concerns for surgery patients appeared present at only rare levels in this sample. The low to very low rates of smoking, substance abuse, and severe mental illness (e.g., schizophrenia, bipolar) reported here, for example, suggest that these factors had limited practical value for discriminating among bariatric candidates. Similarly, psychosocial factors present at low rates are unlikely to provide substantial explanatory power for predicting weight-related bariatric outcomes in the majority of patients completing surgery. This conclusion contrasts with surveys of 
bariatric practice [1], in which factors such as the latter are listed as among the most common psychiatric and behavior contraindicators for surgery, suggesting that they are believed to have strong importance at the stage of bariatric surgery consideration. Combined with the absence of consistent relationships between bariatric outcomes and psychosocial factors demonstrated in previous reviews [e.g., 9, 10], the data presented here suggest that it may be a more effective strategy to focus on specific behaviors linked to effective weight loss practices (e.g., diet and exercise compliance, ability to maintain food and exercise logs, etc.) that may be present at higher rates among some patients with psychiatric disorders, as opposed to measuring surgery readiness based simply on the presence of the psychiatric diagnosis itself.

The low rate of common psychiatric contraindicators we observed is not unheralded. For example, Kalarchian and colleagues [18] observed rates of $1.7 \%$ for current diagnoses of substance abuse and bipolar depression using a structured interview to assess a pre-bariatric sample, values comparable to those reported herein. The latter study also showed that depression (10.4\%), anxiety (24.0\%), and eating disorders such as binge eating disorder $(16.0 \%)$ were the most common psychiatric conditions. This pattern paralleled the results observed here, with the noteworthy difference that we assessed only the frequency of binge eating, not the functional impact or loss of control dimensions important to a binge eating disorder diagnosis. The absence of the latter content likely explains the much higher rates of binge eating endorsed by the current sample compared to previous studies [18, 19].

Although the prevailing perspective is that the presence of psychiatric comorbidities place patients at risk of poorer bariatric surgery outcomes, considerable literature suggests that psychiatric distress is reduced following bariatric surgery (e.g., [20-22]). Based upon the consistently observed improvement in psychiatric status in postbariatric populations, along with the comparable lack of evidence linking pre-bariatric psychiatric status to weightrelated surgical outcomes, some authors have proposed that comorbid psychiatric illness should be considered as reason for rather than against proceeding with bariatric surgery [13]. It is likewise interesting to note that psychiatric conditions and psychosocial factors are poor predictors of weight loss among non-surgical as well as surgical weight loss patients. For example, reviews of psychological predictors of behavioral weight loss interventions (e.g., [23]) show that pre-treatment status on factors such as depression, anxiety, and binge eating, among others, does not consistently predict weight change. If rates of severe psychiatric disorders and substance abuse are rare as shown in this and some previous studies, and the more common mood disorders and eating disorder behaviors are not only unrelated to weight changes but likely to improve as a result of efficacious weight interventions, then our results may be viewed as additional evidence encouraging a reevaluation of the way in which the information collected during pre-bariatric psychological assessments it utilized.

A number of clinically useful alternatives exist for which pre-bariatric data could be employed. For example, mental health factors are common predictors of treatment attrition in weight loss programs [24, 25], and clinicians could use these data to identify those patients who could benefit from efforts to encourage participation in follow-up, support groups, or complimentary diet and exercise interventions accompanying surgery. Psychosocial data may more effectively predict longer-term weight loss or maintenance $[26,27]$ rather than the shorter-term follow-ups common in much of the previous literature. In addition, pre-bariatric psychological data could function as a baseline assessment, from which clinicians can identify both those showing improvement in psychiatric distress over follow-up visits and (perhaps more importantly) those that are not, aiding efforts to recognize those patients warranting additional interventions to promote improvements in function and quality of life.

\section{Limitations}

A number of limitations warrant mention. Psychiatric and biomedical diagnoses were based on patient's self-report on the MOVE!23 questionnaire. The MOVE!23 instrument is a single measure assessing a broad array of medical, psychiatric, and behavioral factors, but contains only single item to several item assessments of any specific area of functioning. As such, MOVE!23 findings are not equivalent to longer psychological questionnaires or interview diagnoses. Although this instrument is a widely used tool developed by NCP specifically for overweight and obese populations [16], additional research is required to demonstrate psychometric support for the MOVE!23 questionnaire, and the MOVE!23 information (excluding height, weight, and BMI, which were physically measured) collected in this study was not confirmed by validated questionnaires, diagnostic interviews, or physical examinations. It is also possible that bariatric candidates in this study were comparatively under reporting symptoms in order to present as more favorable surgery prospects in the future; however, we consider this risk unlikely as the intake class setting serving as the basis of this study is designed to enroll patients in a non-surgical weight management intervention and surgery treatments are not even discussed. We were unable to determine specific reasons why patients did or did not pursue surgery. Although financial costs and lack of insurance coverage is a common barrier to bariatric surgery for many patients, veterans approved for bariatric surgery through VASDHS are not charged for the procedure. The reported sample is also not necessarily representative of the broader Veteran population with obesity, as those described here were treatment seeking 
as defined by their voluntary attendance to the MOVE intake class and, thus, may have been more highly motivated than non-participating veterans with comparable BMI values. Similarly, many — if not the majority [28] — of veterans do not receive their medical care through the VA.

\section{Summary}

In a prospective cohort of bariatric surgery-eligible (BMI $\geq 35)$ patients enrolling in a multidisciplinary weight management program, approximately $25 \%$ went on to bariatric surgery candidacy in the subsequent 2 years. The subgroup of bariatric surgery candidates did not differ on characteristics such as BMI, age, motivation, binge eating, or weight loss expectations and endorsed low rates of severe mental illness. However, bariatric candidates reported lower rates of depression and smoking, fewer cardiovascular risk factors, greater self-rated health, and more problems with obsessive compulsive disorder symptoms. The current results offer an atypical perspective of tracking a cohort of patients over an extended interval to assess factors that differentiate bariatric candidates from other patients seeking treatment for obesity. These results suggest that typical pre-bariatric psychological assessments may be affected by the natural selection process of patients pursuing bariatric surgery, potentially limiting their role as predictors of bariatric outcomes.

Conflict of Interest Statement The authors declare that they have no conflict of interest. No grant support was employed in the completion of the reported study.

Open Access This article is distributed under the terms of the Creative Commons Attribution Noncommercial License which permits any noncommercial use, distribution, and reproduction in any medium, provided the original author(s) and source are credited.

\section{Appendix}

MOVE! 23 (MOVE! Questionnaire)

Please complete the following questionnaire.

All information is confidential and subject to applicable laws regarding privacy of patient records.

1. I consider myself to be (check one):
a. Underweight for my height and age
b. Normal weight for my height and age
c. Overweight for my height and age

2. In general, would you say that your health is (check one):
a. Excellent
b. _ Very Good
c. Good
d. Fair
e. Poor

3. Please indicate (with a check mark to the left) any of the following that apply to you:

Shortness of breath at rest

Chest pains not previously evaluated by your physician

Active infection of any kind

Hernia in the groin or belly area

Retinal hemorrhage (bleeding in the back of

the eye)

$$
\begin{aligned}
& \text { Loss of balance because of dizziness or } \\
& \text { passing out }
\end{aligned}
$$

Any chronic medical problem that has recently

been out-of-control, unstable or flared up

Arthritis or joint pain

Back pain or spinal disc disease

Osteoporosis or bone disease

Amputation

Spinal cord injury

Lung disease such as emphysema, COPD, or asthma

Heart disease such as heart failure, heart attack or angina, heart surgery or angioplasty, irregular heartbeat, implanted defibrillator or pacemaker, heart valve problems

Poor blood circulation in the legs

Stroke or TIAs (mini-strokes) or carotid artery surgery in the neck

Diabetes even if controlled by medication or diet

High blood pressure -even if controlled by medication or diet

High blood cholesterol -even if controlled by medication or diet

Someone in your immediate family with heart problems at an age younger than 50

None of the above

Please indicate any of the following that apply to you:

Too much stress

General unhappiness

Depression

Anxiety problems or nervousness

Family or relationship problems

Bipolar disorder (Manic depressive disorder)

Schizophrenia

Post traumatic stress disorder (PTSD)

Obsessive/compulsive disorder

Eating disorder/binge eating/anorexia/bulimia Tobacco Use/Smoking

Substance Abuse or Dependence

None of these 
4. Have you tried to lose weight in the past? (Circle one.) Yes No

If so, what of the following options have you tried in order to lose weight?

Check all that apply.

a. Some form of dieting, that is eating differently from the way you usually eat for the sake of losing weight

b. __ Avoiding particular foods or food groups

c. Physical exercise, such as walking, swimming or calisthenics

d. Prepackaged meals

e. Meal replacements in bar, powder, liquid, tablet/pill or water form

f. __ Fasting for 24 hours or longer

g. Skipping meals

h. Hypnosis

i. Comprehensive weight loss program with dietary changes, physical activity, and behavioral counseling

j. __ Any other kind of weight loss program that does NOT provide comprehensive treatment (dietary changes, physical activity, and behavioral counseling)

k. Keeping a $\log$ or journal for eating or exercise

1. _ Causing yourself to vomit after you eat

$\mathrm{m}$. Cosmetic procedure such as liposuction or other

n. Weight loss medical procedure such as gastric bypass, gastric banding, wiring of your jaw or other

o. __ Taking a prescription medication to lose weight

p. Taking an over the counter (OTC) medication; vitamin, mineral, or nutrient supplement; herbal supplement; naturopathic or alternative medicine preparation or supplement to lose weight

q. Smoking to control weight

r. Other

5. Are you trying to lose weight now? (Circle one.) Yes No

If so, what does your current weight loss plan include? Check all that apply.

a. __ Some form of dieting, that is eating differently from the way you usually eat for the sake of losing weight

b. __ Avoiding particular foods or food groups

c. __ Physical exercise, such as walking, swimming or calisthenics

d. _ Prepackaged meals

e. Meal replacements in bar, powder, liquid, tablet/pill or water form f. Fasting for 24 hours or longer

g. Skipping meals

h. Hypnosis

i. Comprehensive weight loss program with dietary changes, physical activity, and behavioral counseling

j. ___ Any other kind of weight loss program that does NOT provide comprehensive treatment (dietary changes, physical activity, and behavioral counseling)

k. Keeping a $\log$ or journal for eating or exercise

1. Causing yourself to vomit after you eat

m. Cosmetic procedure such as liposuction or other

n. Weight loss medical procedure such as gastric bypass, gastric banding, wiring of your jaw or other

o. _ Taking a prescription medication to lose weight

p. __ Taking an over the counter (OTC) medication; vitamin, mineral, or nutrient supplement; herbal supplement; naturopathic or alternative medicine preparation or supplement to lose weight

q. Smoking to control weight

r. Other

6. Select the answer that best describes your rate of weight gain over the years.

a. I have been overweight since childhood (before age 18).

b. I have gained weight gradually over the years.

c. I I have gained most of my excess weight in a short period of time.

d. I I have gained and lost weight many times over the years ( yo-yo ).

7. Select the answer that best describes your family:

a. As a group, my family is not overweight or obese.

b. As a group, some members of my family are overweight or obese.

c. As a group, most members of my family are overweight or obese.

8. How much can you rely on family or friends for support and encouragement? (Check one.)
a. A lot
b. Somewhat
c. Not at all

9. How important is controlling your weight to you personally? Please circle the number that applies. Please do not place a circle in the space between numbers.

012345678910

Not Very 
Important Important

At All

10. How confident are you that you can successfully change your eating and physical activity to control your weight? Please circle the number that applies. Please do not place a circle in the space between numbers.

\section{0 \\ Not Very \\ Confident Confident \\ At All}

11. Check the statement that most closely applies to you:

a. I am not considering trying to control my weight at this time.

b. I I considering trying to control my weight sometime within the next six months.

c. I I am ready to make some changes to control my weight.

d. I I am actively working on controlling my weight at this time.

e. I have been continuously and successfully doing things to control my weight for more than the last six months.

12. How much weight do you think you realistically could lose in one year? (Check one.)
a. $10 \mathrm{lbs}$ or less
b. $1125 \mathrm{lbs}$
c. $2650 \mathrm{lbs}$
d. $51-100 \mathrm{lbs}$
e. more than $100 \mathrm{lbs}$

13. How satisfied are you with the appearance of your body? (Check one.)
a. Very satisfied
b. Moderately satisfied
c. Neither satisfied or dissatisfied
d. Moderately dissatisfied
e. Very dissatisfied

14. Do any of the following have anything to do with your being overweight?

Check all that apply to you.
a. Eating because of emotions or stress
b. Family or relationship problems
c. Boredom
d. Loneliness or Loss of loved one
e. Eating too much
f. Poor food choices or habits
g. Not getting enough physical activity
h. Difficulty with self control
i. Hungry all the time
j. __ Feeling bad about myself
k. Love to eat
1. Quitting tobacco use
m. Pregnancy/Childbirth
n. Illness or injury
o. Medications led to weight gain
p. Other
q. None of the above

15. What do you think may get in the way of changing your eating habits?

Check all that apply to you.

a. __ Eating food from restaurants, fast food places, convenience stores, vending machines

b. Person who prepares my food is uncooperative or unsupportive

c. Too much high calorie food available at home or work

d. _ Too little time to prepare and eat healthy food

e. Too little money to buy healthy food

f. Feeling hungry much of the time

g. Used to eating a certain way

h. Difficulties such as stress or depression

i. Being with others who overeat

j. _ Dont know how

k. Other

1. Nothing should get in the way

16. How many times a day do you typically eat, including snacks? (Check one.)
a. 1 time a day
b. 2 times a day
c. 3 times a day
d. 4 times a day
e. 5 or more times each day

17. How many times per week do you eat at restaurants or buy take out food?

Please indicate on the line below the number of times between 0 and 21 .

Consider breakfast, lunch and supper 7 days a week for a total of 21 meals for which restaurant or take out food could be eaten.

When you eat out, do you find that you overeat or eat higher calorie foods?
a. — Yes
b. $\quad$ No

18. How much juice (including juice-drinks) or sugarsweetened soda, tea or other beverages do you drink most days? (Check one option below.)

a. I I don t drink juice; juice-drinks; or sugarsweetened soda, tea or other beverages.

b. 12 cups, cans, small bottles or drink boxes per day

c. 3 or more cups, cans, small bottles or drink boxes per day 
19. Do you drink alcoholic beverages (such as beer, malt liquor, wine, wine coolers, hard/distilled liquor)? (Check one.)
a. $\longrightarrow$ Yes
b. $\quad$ No

20. How fast do you usually eat? (Check one.)
a. I eat slowly.
b. I eat at a moderate pace.
c. I eat fast.

21. On average, how often have you eaten extremely large amounts of food at one time and felt that your eating was out of control at that time? (Check one.)
a. Never
b. Less than 1 time per week
c. 1 time per week
d. 2 to 4 times a week
e. 5 or more times a week

22. What do you think may get in the way of changing your physical activity habits?

Check all that apply to you.
a. Too little time
b. Too little money
c. Safety concerns
d. No place to walk or be active
e. No transportation
f. Lack of support or encouragement from others
g. Difficulties such as stress, depression, etc.
h. Do not like to exercise
i. Daily habits or routines that do not include exercise
j. Pain
k. Amputation
1. Back problems
m. Arthritis
n. Muscular problems
o. Heart or lung disease
p. Joint problems
q. Spinal cord injury
r. Too tired
s. Job or work schedule
t. Other
u. Nothing should get in the way

23. This next question asks about your physical activity habits.

There are two types of activity to consider:

Moderate physical activities cause light sweating and a slight to moderate increase in breathing or heart rate. Examples include brisk walking, bicycling, vacuuming, gardening, and golfing without a cart.
Vigorous activities cause heavy sweating and large increases in breathing or heart rate. Examples include running, aerobic classes, heavy yard work, and briskly swimming laps.

a. How many days per week do you do moderate activities for at least 10 minutes at a time? Please circle the appropriate number. Please do not place a circle in the space between numbers.

$$
01234567
$$

b. On days when you do moderate activities for at least 10 minutes at a time, how much total time per day do you spend doing these activities? (Select one choice below.)
a. 10-19 minutes
b. 20-29 minutes
c. 30-59 minutes
d. _ > $>60$ minutes

c. How many days per week do you do vigorous activities for at least 10 minutes at a time? Please circle the appropriate number. Please do not place a circle in the space between numbers.

$$
01234567
$$

d. On days when you do vigorous activities for at least 10 minutes at a time, how much total time per day do you spend doing these activities? (Select one choice below.)
a. 10-19 minutes
b. 20-29 minutes
c. 30-59 minutes
d. $>=60$ minutes

\section{References}

1. Bauchowitz AU, Gonder-Frederick LA, Olbrisch ME, et al. Psychosocial evaluation of bariatric surgery candidates: a survey of present practices. Psychosom Med. 2005;67(5):82532.

2. Fabricatore AN, Crerand CE, Wadden TA, et al. How do mental health professionals evaluate candidates for bariatric surgery? Survey results. Obes Surg. 2006;16:567-73.

3. LeMont D, Moorehead MK, Parish MS, Reto CS, Ritz SJ. Suggestions for the pre-surgical psychological assessment of bariatric surgery candidates. Available at: http://www.asbs.org/ html/pdf/PsychPreSurgicalAssessment.pdf. Accessed 5 April 2010.

4. Mühlhans B, Horbach T, de Zwaan M. Psychiatric disorders in bariatric surgery candidates: a review of the literature and results of a German prebariatric surgery sample. Gen Hosp Psychiatry. 2009;31:414-21.

5. Wadden TA, Sarwer DB. Behavioral assessment of candidates for bariatric surgery: a patient-oriented approach. Surg Obes Relat Dis. 2006;2:171-9.

6. Pull CB. Current psychological assessment practices in obesity surgery programs: what to assess and why. Curr Opin Psychiatry. 2010;23(1):30-6. 
7. Blackburn GL, Hutter MM, Harvey AM, et al. Expert panel on weight loss surgery: executive report update. Obesity. 2009; 17:842-62.

8. van Hout GC, Verschure SK, van Heck GL. Psychosocial predictors of success following bariatric surgery. Obes Surg. 2005;15(4):552-60.

9. Herpertz S, Kielmann R, Wolf AM, et al. Do psychosocial variables predict weight loss or mental health after obesity surgery? A systematic review. Obes Res. 2004;12(10):1554-69.

10. Greenberg I, Perna F, Kaplan M, et al. Behavioral and psychological factors in the assessment and treatment of obesity surgery patients. Obes Res. 2005;13(2):244-9.

11. Bocchieri LE, Meana M, Fisher BL. A review of psychosocial outcomes of surgery for morbid obesity. J Psychosom Res. 2002;52:155-65.

12. Sarwer DB, Wadden TA, Fabricatore AN. Psychosocial and behavioral aspects of bariatric surgery. Obes Res. 2005;13:639-48.

13. Simon GE, Arterburn DE. Does comorbid psychiatric disorder argue for or against surgical treatment of obesity? Gen Hosp Psychiatry. 2009;31:401-2.

14. Santry HP, Chin MH, Cagney KA, et al. The use of multidisciplinary teams to evaluate bariatric surgery patients: results from a national survey in the U.S.A. Obes Surg. 2006;16:59-66.

15. Sackett PR, Yang H. Correction for range restriction: an expanded typology. J Appl Psychol. 2000;85:112-8.

16. Kinsinger LS, Jones KR, Kahwati L, Harvey R, Burdick M, Zele $\mathrm{V}$, Yevich SJ. Design and dissemination of the MOVE! weightmanagement program for veterans. Prev Chronic Dis 2009;6(3). http://www.cdc.gov/pcd/issues/2009/jul/08_0150.htm. Accessed 12 Jul 2010

17. Walfish S, Vance D, Fabricatore AN. Psychological evaluation of bariatric surgery applicants: procedures and reasons for delay or denial of surgery. Obes Surg. 2007;17:1578-83.
18. Kalarchian MA, Marcus MD, Levine MD, et al. Psychiatric disorders among bariatric surgery candidates: relationship to obesity and functional health status. Am J Psychiatry. 2007;164:328-34.

19. Bocchieri-Ricciardi LE, Chen EY, Munoz D, et al. Pre-surgery binge eating status: effect on eating behavior and weight outcome after gastric bypass. Obes Surg. 2006;16:1198-204.

20. Niego SH, Kofman MD, Weiss JJ, et al. Binge eating in the bariatric surgery population: a review of the literature. Int J Eat Disord. 2007;40:349-59.

21. Dymek MP, le Grange D, Neven K, et al. Quality of life and psychosocial adjustment in patients after Roux-en-Y gastric bypass: a brief report. Obes Surg. 2001;11:32-9.

22. Thonney B, Pataky Z, Badel S, et al. The relationship between weight loss and psychosocial functioning among bariatric surgery patients. Am J Surg. 2010;199:183-8.

23. Teixeira PJ, Going SB, Sardinha LB, et al. A review of psychosocial pre-treatment predictors of weight control. Obes Rev. 2005;6:43-65.

24. Fabricatore AN, Wadden TA, Moore RH, et al. Predictors of attrition and weight loss success: results from a randomized controlled trial. Behav Res Ther. 2009;47:685-91.

25. Teixeira PJ, Going SB, Houtkooper LB, et al. Pretreatment predictors of attrition and successful weight management in women. Int J Obes. 2004;28:1124-33.

26. Wing RR, Phelan S. Long-term weight loss maintenance. Am J Clin Nutr. 2005;82:222S-5.

27. Kinzl JF, Schrattenecker M, Traweger C, et al. Psychosocial predictors of weight loss after bariatric surgery. Obes Surg. 2006;16:1609-14.

28. Kaplan MS, Huguet N, McFarland BH, et al. Suicide among male veterans: a prospective population-based study. J Epidemiol Community Health. 2007;61(7):619-24. 